\title{
Heterotic compactifications on nearly Kähler manifolds
}

\author{
Olaf Lechtenfeld, ${ }^{a, b}$ Christoph Nölle ${ }^{a}$ and Alexander D. Popov ${ }^{c}$ \\ ${ }^{a}$ Institut für Theoretische Physik, Leibniz Universität Hannover, \\ Appelstraße 2, 30167 Hannover, Germany \\ ${ }^{b}$ Centre for Quantum Engineering and Space-Time Research, Leibniz Universität Hannover, \\ Welfengarten 1, 30167 Hannover, Germany \\ ${ }^{c}$ Bogoliubov Laboratory of Theoretical Physics, JINR, \\ 141980 Dubna, Moscow Region, Russia \\ E-mail: lechtenf@itp.uni-hannover.de, noelle@itp.uni-hannover.de, \\ popov@theor.jinr.ru
}

ABSTRACT: We consider compactifications of heterotic supergravity on anti-de Sitter space, with a six-dimensional nearly Kähler manifold as the internal space. Completing the model proposed by Frey and Lippert [10] with the particular choice of $\mathrm{SU}(3) / \mathrm{U}(1) \times \mathrm{U}(1)$ for the internal manifold, we show that it satisfies not only the supersymmetry constraints but also the equations of motion with string corrections of order $\alpha^{\prime}$. Furthermore, we present a nonsupersymmetric model. In both solutions we find confirmed a recent result of Ivanov [18] on the connection used for anomaly cancellation. Interestingly, the volume of the internal space is fixed by the supersymmetry constraints and/or the equations of motion.

Keywords: Flux compactifications, Superstring Vacua, Differential and Algebraic Geometry

ArXiv EPRINT: 1007.0236 


\section{Contents}

1 Introduction $\quad 1$

2 Action, supersymmetry and field equations 2

$\begin{array}{lll}3 & \text { Nearly Kähler manifolds } & 7\end{array}$

4 Supersymmetric solutions with gaugino condensate $\quad 13$

5 Non-supersymmetric solutions with vanishing gaugino 17

$\begin{array}{llr}6 & \text { Conclusions } & 18\end{array}$

\section{Introduction}

There has been considerable interest in heterotic flux compactifications in recent years. Compared to the traditional Calabi-Yau models with their problematic moduli, these might include more realistic examples where all moduli are fixed by a steep effective potential. A pioneering work in this direction was Strominger's analysis of supersymmetric compactifications with only bosonic fields non-vanishing [24], leading to his well-known set of conditions on the internal manifold and the fields. Although it has been known for quite some time now that these conditions admit non-trivial solutions [20], explicit models are rare $[9,12,25]$.

It has been proposed in $[10,13]$ to consider more general vacua with external antide Sitter space, where the gaugino is not supposed to vanish. Similarly to Strominger's approach Frey and Lippert use the condition for supersymmetric vacua to derive constraints on the internal manifold [10]. Among their solutions the simplest models are given by a direct product of four-dimensional anti-de Sitter space and a six-dimensional compact nearly Kähler manifold. Nearly Kähler manifolds carry an SU(3) structure [8, 11] with totally antisymmetric intrinsic torsion, giving rise to a three-form. The latter makes them promising candidates for compactification models where one can take the $H$-field equal to the intrinsic torsion. This relation follows indeed from the supersymmetry conditions. Furthermore, it is suspected that nearly Kähler compactifications have few moduli; e.g. only four compact six-dimensional nearly Kähler spaces are known.

The model proposed by Frey and Lippert is incomplete, since their discussion of the Bianchi identity assumes a certain form of the gauge field, which does not have the instanton property necessary for the vanishing of the gaugino variation. This problem has already been adressed by Manousselis, Prezas and Zoupanos [21], who also include a dilatino condensate in their considerations, to have more freedom to adjust parameters. They showed that there exist complete supersymmetric solutions for all of the four nearly Kähler spaces. 
Anomaly cancellation in heterotic string theory requires the introduction of a connection $\tilde{\Gamma}$ on the tangent bundle. One usually takes the Levi-Cività connection plus a torsion given by the $H$-field, yet other choice are possible. For bosonic Minkowski compactifications, Ivanov has shown recently that the choice of $\tilde{\Gamma}$ crucially determines whether or not the equations of motion follow from the supersymmetry conditions and the Bianchi identity for $H$ [18]. We find this result confirmed: supersymmetric solutions fulfil the dilaton equation only if $\tilde{\Gamma}$ is the instanton connection on the internal space.

In this paper, we construct two complete solutions to the heterotic supergravity equations with string corrections of order $\alpha^{\prime}$, on the space $\operatorname{AdS}_{4}(r) \times K(\rho)$, where $K(\rho)$ is the nearly Kähler coset $\mathrm{SU}(3) / \mathrm{U}(1) \times \mathrm{U}(1)$ with scale $\rho$. We restrict ourselves to a vanishing gravitino and dilatino, ${ }^{1}$ which has the advantage that we are able to check explicitly whether the equations of motion are satisfied. Although generally one expects the vanishing of the fermionic supersymmetry variations to imply the equations of motion, this is not always the case. Our first solution is supersymmetric and features a gaugino condensate, while our second one is non-supersymmetric and without gaugino.

After presenting the action, supersymmetry transformation and field equations in section 2, we find the AdS radius $r$ fixed in terms of $\alpha^{\prime}$. The rich differential geometry of homogeneous nearly Kähler spaces is reviewed in section 3, where we also calculate all the quantities appearing in the equations throughout this paper. In section 4 we combine the constraints on the external and internal geometries and show that at least the space $\mathrm{SU}(3) / \mathrm{U}(1) \times \mathrm{U}(1)$ supports supersymmetric models, which are not contained in the solutions of [21]. In section 5 we present a non-supersymmetric solution to all equations of motion (and the Bianchi identity), again on the coset $\mathrm{SU}(3) / \mathrm{U}(1) \times \mathrm{U}(1)$, with vanishing gaugino for simplicity. Again for $\tilde{\Gamma}$ we need to choose the instanton connection. In both solutions, the gauge connection is proportional to the spin connection, and the scale $\rho$ of the internal manifold is determined by $\alpha^{\prime}$. In the Conclusions we combine our results and display the explicit form of our two solutions. Attempts to solve the equations for the alternative $K=\mathrm{SU}(2)^{3} / \mathrm{SU}(2)$ require a different choice for $\tilde{\Gamma}$, which fails to satisfy the dilaton equation.

\section{Action, supersymmetry and field equations}

Field content. The low-energy field theory limit of heterotic string theory is given by $d=10, \mathcal{N}=1$ supergravity coupled to a super-Yang-Mills multiplet. It contains the following fields living on the $10 d$ spacetime $M$ and transforming in particular irreps of the tangent $\mathrm{SO}(9,1)[14]$ :

- graviton $g$, a metric on $M$, in a $\underline{54}$

- dilaton $\phi$, a function on $M$, in a $\underline{1}$

- Kalb-Ramond field $B$, a two-form on $M$, in a $\underline{45}$

\footnotetext{
${ }^{1}$ In the equations of motion, we also put the dilaton to zero.
} 
- gauge field $A$, a one-form on $M$, in a $\underline{10}$

- gravitino $\psi$, left-handed Majorana-Weyl vector-spinor, in a $\underline{144}_{s}$

- dilatino $\lambda$, right-handed Majorana-Weyl spinor, in a $\underline{16}_{s}$

- gaugino $\chi$, left-handed Majorana-Weyl spinor, in a $\underline{16}_{c}$

Furthermore there is a left-handed Majorana-Weyl supersymmetry generator $\varepsilon$. Besides these fundamental fields, we need the curvature forms

$$
F=d A+A \wedge A \quad \text { and } \quad H=d B+\frac{1}{4} \alpha^{\prime}\left[\omega_{C S}(\tilde{\Gamma})-\omega_{C S}(A)\right]
$$

where $\omega_{C S}$ denotes the Chern-Simons-forms

$$
\omega_{C S}(\tilde{\Gamma})=\operatorname{tr}\left(\tilde{R} \wedge \tilde{\Gamma}-\frac{2}{3} \tilde{\Gamma} \wedge \tilde{\Gamma} \wedge \tilde{\Gamma}\right) \quad \text { and } \quad \omega_{C S}(A)=\operatorname{tr}\left(F \wedge A-\frac{2}{3} A \wedge A \wedge A\right)
$$

and $\tilde{\Gamma}$ is a connection on $T M$, whose choice is ambiguous. ${ }^{2}$ Basic choices are:

- the Levi-Cività connection $\Gamma^{\mathrm{LC}}(g)$

- the plus-connection $\Gamma^{+}=\Gamma^{\mathrm{LC}}-\frac{1}{2} H$

- the minus-connection $\Gamma^{-}=\Gamma^{\mathrm{LC}}+\frac{1}{2} H$

- the Chern connection $\Gamma^{\mathrm{Ch}}[24]$.

Finally, there appears the space-time curvature form

$$
\tilde{R}=d \tilde{\Gamma}+\tilde{\Gamma} \wedge \tilde{\Gamma}
$$

for whichever connection has been chosen. Traces are taken over the adjoint representation of the gauge group or of $\mathrm{SO}(9,1)$, depending on the context.

Action and supersymmetry. In this paper we are putting the gravitino and dilatino to zero,

$$
\psi=0 \quad \text { and } \quad \lambda=0 .
$$

With this, the low-energy action up to and including terms of order $\alpha^{\prime}$ reads $[4,10]$

$\mathcal{S}(g, \phi, B, \chi, A)=\int_{M} d^{10} x \sqrt{\operatorname{det} g} e^{-2 \phi}\left\{\mathrm{Scal}+4|d \phi|^{2}-\frac{1}{2}|T|^{2}+\frac{1}{4} \alpha^{\prime} \operatorname{tr}\left(|\tilde{R}|^{2}-|F|^{2}-2 \bar{\chi} \mathcal{D} \chi\right)\right\}$,

where

$$
T=H-\frac{1}{2} \Sigma \quad \text { with } \quad \Sigma=\frac{1}{24} \alpha^{\prime} \operatorname{tr}\left(\bar{\chi} \gamma_{M} \gamma_{N} \gamma_{P} \chi\right) d x^{M} \wedge d x^{N} \wedge d x^{P}
$$

\footnotetext{
${ }^{2}$ Different connections correspond to different regularization schemes in the $2 d$ sigma model, and are related by field redefinitions, see [17] and the list of references for different choices in the introduction of [9].
} 
and

$$
\operatorname{tr}|\tilde{R}|^{2}=\frac{1}{2} \tilde{R}_{M N P Q} \tilde{R}^{M N P Q} \quad \text { and } \quad \operatorname{tr}|F|^{2}=\frac{1}{2} \operatorname{tr} F_{M N} F^{M N}
$$

Finally, $\mathcal{D}=\gamma^{M} \nabla_{M}$ denotes the Dirac operator, coupled to $\Gamma^{\mathrm{LC}}(g)$ and to $A$. The action is invariant under $\mathcal{N}=1$ supersymmetry transformations [4], which act on the fermions as

$$
\begin{aligned}
\delta \psi_{M} & =\nabla_{M} \varepsilon-\frac{1}{8} H_{M N P} \gamma^{N} \gamma^{P} \varepsilon+\frac{1}{96} \gamma(\Sigma) \gamma_{M} \varepsilon \\
\delta \lambda & =-\frac{1}{2} \gamma\left(d \phi-\frac{1}{12} H-\frac{1}{48} \Sigma\right) \varepsilon \\
\delta \chi & =-\frac{1}{4} \gamma(F) \varepsilon
\end{aligned}
$$

where $\gamma$ denotes the map from forms to the Clifford algebra,

$$
\gamma\left(\frac{1}{p !} \omega_{M_{1} \ldots M_{p}} d x^{M_{1}} \wedge \cdots \wedge d x^{M_{p}}\right)=\omega_{M_{1} \ldots M_{p}} \gamma^{M_{1}} \ldots \gamma^{M_{p}}
$$

Field equations. The equations of motion take the form (we symmetrize with weight one)

$$
\begin{aligned}
\operatorname{Ric}_{M N}+2(\nabla d \phi)_{M N}-\frac{1}{8} T_{P Q(M} H_{N)}{ }^{P Q}+ & \\
+\frac{1}{4} \alpha^{\prime}\left[\tilde{R}_{M P Q R} \tilde{R}_{N}^{P Q R}-\operatorname{tr}\left(F_{M P} F_{N}{ }^{P}-\frac{1}{2} \bar{\chi} \gamma_{(M} \nabla_{N)} \chi\right)\right] & =0, \\
\text { Scal }+4 \Delta \phi-4|d \phi|^{2}-\frac{1}{2}|T|^{2}+\frac{1}{4} \alpha^{\prime} \operatorname{tr}\left[|\tilde{R}|^{2}-|F|^{2}-2 \bar{\chi} \mathcal{D} \chi\right] & =0, \\
\left(\mathcal{D}-\frac{1}{24} \gamma(T)\right) e^{-2 \phi} \chi & =0, \\
e^{2 \phi} d *\left(e^{-2 \phi} F\right)+A \wedge * F-* F \wedge A+* T \wedge F & =0, \\
d * e^{-2 \phi} T & =0 .
\end{aligned}
$$

The derivation of the equations is greatly simplified by a Lemma in [4], as was pointed out by Becker and Sethi [3]. It implies that up to this order in $\alpha^{\prime}$ one can neglect variations of the form $\frac{\partial S}{\partial \tilde{\Gamma}} \frac{\partial \tilde{\Gamma}}{\partial(\cdots)}$, for any field $(\cdots)$. Besides these equations, the Bianchi identity for $H$ must be satisfied, which follows from the definition (2.1):

$$
d H=\frac{1}{4} \alpha^{\prime} \operatorname{tr}[\tilde{R} \wedge \tilde{R}-F \wedge F] .
$$

One expects the vanishing of the fermionic supersymmetry transformations plus the Bianchi identity to imply all equations of motion, but whether or not this is true depends on the connection $\tilde{\Gamma}^{3}$

\footnotetext{
${ }^{3}$ Ivanov [18] proves that solutions of Strominger's equations (which are equivalent to the vanishing of (2.8) with vanishing gaugino) satisfy the equations of motion only for $\tilde{\Gamma}=\Gamma^{-}$.
} 
Taking the trace of the Einstein equation (the first one in (2.10)) gives

Scal $+2 \Delta \phi-\frac{3}{2}(T, H)+\frac{1}{2} \alpha^{\prime} \operatorname{tr}\left[|\tilde{R}|^{2}-|F|^{2}-\frac{1}{2} \bar{\chi} \mathcal{D} \chi\right]=0 \quad$ with $\quad(T, H) \equiv \frac{1}{3 !} T_{M N P} H^{M N P}$.

This equation can be combined with the dilaton equation (the second one in (2.10)) in two different ways as

$$
\begin{aligned}
e^{2 \phi} \Delta e^{-2 \phi}+\frac{1}{2}|T|^{2}-\frac{3}{2}(T, H)+\frac{1}{4} \alpha^{\prime} \operatorname{tr}\left[|\tilde{R}|^{2}-|F|^{2}+\bar{\chi} \mathcal{D} \chi\right] & =0 \\
\text { Scal }-\frac{9}{2} e^{4 \phi / 3} \Delta e^{-4 \phi / 3}-|T|^{2}+\frac{3}{2}(T, H)-\frac{3}{4} \alpha^{\prime} \operatorname{tr}(\bar{\chi} \mathcal{D} \chi) & =0 .
\end{aligned}
$$

One can replace the dilaton equation by one these, and if further the Einstein equation is satisfied, then the other equation in (2.13) is implied. For the remainder of the paper we put the dilaton to zero,

$$
\phi=0
$$

motivated by the results in [10]. This noticeably simplifies the equations of motion (2.10).

Space-time factorization. Our interest is in space-time manifolds of direct product form,

$$
M=\operatorname{AdS}_{4}(r) \times K,
$$

with a $4 d$ anti-de Sitter space of 'radius' $r$ as 'external' factor and a $6 d$ compact Riemannian 'internal' space $K$. Small Greek indices shall be restricted to the external part, while small Latin indices will be reserved for the internal dimensions. Furthermore, we assume that $F, H$ and $\Sigma$ are restricted to $K$, i.e. they do not depend on the AdS coordinates. The components of $\tilde{R}$ in AdS direction are taken to coincide with the Riemann curvature of AdS. This further simplifies the equations. From now on, hatted quantities refer to the AdS part, and unhatted ones live on $K$. The ambiguity in picking $\tilde{\Gamma}$ is a choice of connection on $K$.

As a consequence of the splitting, the equations of motion (2.10) decompose. The Einstein equation (first in (2.10)) splits into

$$
\begin{aligned}
\widehat{\operatorname{Ric}}_{\mu \nu}+\frac{1}{4} \alpha^{\prime} \widehat{R}_{\mu \alpha \beta \gamma} \widehat{R}_{\nu}^{\alpha \beta \gamma} & =\frac{1}{8} \alpha^{\prime} \operatorname{tr}\left(\bar{\chi} \widehat{\gamma}_{(\mu} \widehat{\nabla}_{\nu)} \chi\right), \\
0 & =\frac{1}{8} \alpha^{\prime} \operatorname{tr}\left(\bar{\chi}\left(\widehat{\gamma}_{\mu} \nabla_{a}+\gamma_{a} \widehat{\nabla}_{\mu}\right) \chi\right) \\
\operatorname{Ric}_{a b}-\frac{1}{8} T_{c d(a} H_{b)}{ }^{c d}+\frac{1}{4} \alpha^{\prime}\left[\tilde{R}_{a c d e} \tilde{R}_{b}^{c d e}-\operatorname{tr}\left(F_{a c} F_{b}^{c}\right)\right] & =\frac{1}{8} \alpha^{\prime} \operatorname{tr}\left(\bar{\chi} \gamma_{(a} \nabla_{b)} \chi\right),
\end{aligned}
$$

and the two combinations (2.13) then read

$$
\begin{aligned}
\frac{1}{2}|T|^{2}-\frac{3}{2}(T, H)+\frac{1}{4} \alpha^{\prime} \operatorname{tr}\left[|\widehat{R}|^{2}+|\tilde{R}|^{2}-|F|^{2}\right] & =-\frac{1}{4} \alpha^{\prime} \operatorname{tr}(\bar{\chi}(\widehat{\mathcal{D}}+\mathcal{D}) \chi), \\
\widehat{\text { Scal }}+\text { Scal }-|T|^{2}+\frac{3}{2}(T, H) & =+\frac{3}{4} \alpha^{\prime} \operatorname{tr}(\bar{\chi}(\widehat{\mathcal{D}}+\mathcal{D}) \chi) .
\end{aligned}
$$


The gaugino, Yang-Mills and Kalb-Ramond equations become

$$
\begin{aligned}
\left(\widehat{\mathcal{D}}+\mathcal{D}-\frac{1}{24} \gamma(T)\right) \chi & =0, \\
d * F+A \wedge * F-* F \wedge A+* T \wedge F & =0, \\
d * T & =0 .
\end{aligned}
$$

The gravitational data on $\mathrm{AdS}_{4}(r)$ are

$$
\widehat{\mathrm{Scal}}=-\frac{12}{r^{2}}, \quad \widehat{\mathrm{Ric}}=\frac{1}{4} \widehat{\mathrm{Scal}} \widehat{g}=-\frac{3}{r^{2}} \widehat{g}, \quad \widehat{R}_{\mu \alpha \beta \gamma} \widehat{R}_{\nu}^{\alpha \beta \gamma}=\frac{1}{24} \widehat{\mathrm{Scal}}^{2} \widehat{g}_{\mu \nu}=\frac{6}{r^{4}} \widehat{g}_{\mu \nu} .
$$

The gaugino is taken to factorize as

$$
\chi=\widehat{\chi} \otimes \eta+\widehat{\chi}^{*} \otimes \eta^{*}
$$

where $\widehat{\chi}$ is an anticommuting spinor on $\mathrm{AdS}_{4}$ with values in the adjoint of the gauge group, while $\eta$ denotes a commuting spinor on $K$, which we assume to be normalized: $\bar{\eta} \eta=1$. On $\widehat{\chi}$ we impose the massless Dirac equation on $\mathrm{AdS}_{4}[1]$,

$$
\widehat{\mathcal{D}} \widehat{\chi}=0,
$$

so that the gaugino equation (first in (2.18)) implies

$$
\left(\mathcal{D}-\frac{1}{24} \gamma(T)\right) \eta=0 \quad \text { and } \quad \operatorname{tr}(\bar{\chi} \mathcal{D} \chi)=\frac{1}{\alpha^{\prime}}(T, \Sigma) .
$$

Inserting these relations into the equations of motion, we obtain the conditions ${ }^{4}$

$$
\begin{aligned}
& -\left(\frac{3}{r^{2}}-\frac{3}{2 r^{4}} \alpha^{\prime}\right) \widehat{g}_{\mu \nu}=\frac{1}{8} \alpha^{\prime} \operatorname{tr}\left(\widehat{\bar{\chi}}_{(\mu} \widehat{\nabla}_{\nu)} \widehat{\chi}+\widehat{\bar{\chi}}^{*} \widehat{\gamma}_{(\mu} \widehat{\nabla}_{\nu)} \widehat{\chi}^{*}\right), \\
& 0=\operatorname{tr}\left(\left(\hat{\bar{\chi}} \widehat{\gamma}_{\mu} \widehat{\chi}\right)\left(\bar{\eta} \nabla_{a} \eta\right)+\left(\widehat{\bar{\chi}}^{*} \widehat{\gamma}_{\mu} \widehat{\chi}^{*}\right)\left(\bar{\eta}^{*} \nabla_{a} \eta^{*}\right)-\left(\widehat{\bar{\chi}} \widehat{\nabla}_{\mu} \widehat{\chi}^{*}\right)\left(\bar{\eta} \gamma_{a} \eta^{*}\right)+\left(\widehat{\bar{\chi}}^{*} \widehat{\nabla}_{\mu} \widehat{\chi}\right)\left(\bar{\eta}^{*} \gamma_{a} \eta\right)\right), \\
& \operatorname{Ric}_{a b}-\frac{1}{8} T_{c d(a} H_{b)}{ }^{c d}+\frac{1}{4} \alpha^{\prime}\left[\tilde{R}_{a c d e} \tilde{R}_{b}^{c d e}-\operatorname{tr}\left(F_{a c} F_{b}^{c}\right)\right]= \\
& =\frac{1}{8} \alpha^{\prime} \operatorname{tr}\left(\left(\widehat{\bar{\chi}}^{*} \widehat{\chi}\right)\left(\bar{\eta}^{*} \gamma_{(a} \nabla_{b)} \eta\right)-\left(\widehat{\bar{\chi}} \widehat{\chi}^{*}\right)\left(\bar{\eta} \gamma_{(a} \nabla_{b)} \eta^{*}\right)\right), \\
& \frac{1}{2}|T|^{2}-\frac{3}{2}(T, H)+\frac{3}{r^{4}} \alpha^{\prime}+\frac{1}{4} \alpha^{\prime} \operatorname{tr}\left[|\tilde{R}|^{2}-|F|^{2}\right]=-\frac{1}{4}(T, \Sigma), \\
& -\frac{12}{r^{2}}+\mathrm{Scal}+\frac{1}{2}|T|^{2}=0 \\
& \left(\mathcal{D}-\frac{1}{24} \gamma(T)\right) \eta=0 \\
& d * F+A \wedge * F-* F \wedge A+* T \wedge F=0, \\
& d * T=0 .
\end{aligned}
$$

which entangle the internal fields with the AdS data.

\footnotetext{
${ }^{4}$ Note that $\widehat{\bar{\chi}}=\widehat{\chi}^{\dagger} \gamma^{0}$ but $\bar{\eta}=\eta^{\dagger}$.
} 
On the right-hand side of these equations we encounter external gaugino bilinears, which are nilpotent on the classical level. The standard lore to give meaning to these terms performs a quantum average $\langle\ldots\rangle$ over the space-time fermionic degrees of freedom. At this stage, assumptions about the fermionic quantum correlators enter: we assume the presence of a suitable space-time gaugino condensate as a backdrop for the bosonic equations, namely

$$
\left\langle\operatorname{tr} \widehat{\bar{\chi}} \widehat{\chi}^{*}\right\rangle=i \Lambda^{3} \quad \text { but } \quad\langle\operatorname{tr} \widehat{\widehat{\chi}} \widehat{M} \widehat{\chi}\rangle=\left\langle\operatorname{tr} \widehat{\widehat{\chi}} \widehat{M} \widehat{\chi}^{*}\right\rangle=0
$$

for all non-scalar operators $\widehat{M}$, to be consistent with (2.22). The condensate scale $\Lambda \in \mathbb{R}$ will be fixed later. After averaging over the gaugino, our set of equations (2.23) simplifies to

$$
\begin{aligned}
-\left(\frac{3}{r^{2}}-\frac{3}{2 r^{4}} \alpha^{\prime}\right) \widehat{g}_{\mu \nu} & =0 \\
\operatorname{Ric}_{a b}-\frac{1}{8} T_{c d(a} H_{b)}{ }^{c d}+\frac{1}{4} \alpha^{\prime}\left[\tilde{R}_{a c d e} \tilde{R}_{b}^{c d e}-\operatorname{tr}\left(F_{a c} F_{b}^{c}\right)\right] & =\frac{i}{8} \Lambda^{3} \alpha^{\prime}\left(\bar{\eta}^{*} \gamma_{(a} \nabla_{b)} \eta-\bar{\eta} \gamma_{(a} \nabla_{b)} \eta^{*}\right), \\
\frac{1}{2}|T|^{2}-\frac{3}{2}(T, H)+\frac{3}{r^{4}} \alpha^{\prime}+\frac{1}{4} \alpha^{\prime} \operatorname{tr}\left[|\tilde{R}|^{2}-|F|^{2}\right] & =-\frac{1}{4}(T, \Sigma), \\
-\frac{12}{r^{2}}+\operatorname{Scal}+\frac{1}{2}|T|^{2} & =0, \\
\left(\mathcal{D}-\frac{1}{24} \gamma(T)\right) \eta & =0, \\
d * F+A \wedge * F-* F \wedge A+* T \wedge F & =0, \\
d * T & =0,
\end{aligned}
$$

where we continue to use the symbol $\Sigma$ for the condensate

$$
\langle\Sigma\rangle=\frac{i}{24} \Lambda^{3} \alpha^{\prime}\left(\bar{\eta}^{*} \gamma_{a} \gamma_{b} \gamma_{c} \eta-\bar{\eta} \gamma_{a} \gamma_{b} \gamma_{c} \eta^{*}\right) d x^{a} \wedge d x^{b} \wedge d x^{c} .
$$

Remarkably, the first equation fixes the $\mathrm{AdS}_{4}$ radius in terms of $\alpha^{\prime}$,

$$
r^{2}=\frac{1}{2} \alpha^{\prime}
$$

We note that tracing the second equation simplifies its right-hand side to $\frac{1}{8}(T, \Sigma)$, proportional to the right-hand side of the third equation. In the fourth equation (the dilaton equation), the negative contribution $\widehat{\text { Scal }}=-\frac{12}{r^{2}}$ allows for internal manifolds of positive scalar curvature, which are excluded in the usual Minkowski compactifications. The final three equations are conditions on the commuting spinor $\eta$, the Yang-Mills connection $A$ and the torsion $H$ on the internal manifold $K$. We shall construct solutions to them, after having introduced the geometry of nearly Kähler manifolds in the following section.

\section{$3 \quad$ Nearly Kähler manifolds}

Definitions. A six-dimensional Riemannian manfiold $(K, g)$ is said to carry an $\mathrm{SU}(3)$ structure, if there is a compatible almost-complex structure $J \in \Gamma(\operatorname{End}(T K))$, with $J^{2}=$ 
-1 and $g(J X, J Y)=g(X, Y)$, the associated two-form $\omega=g(\cdot, J \cdot) \in \Omega^{(1,1)}(K)$, and a non-vanishing (3,0)-form $\Omega \in \Omega^{(3,0)}(K)$. Every $\mathrm{SU}(3)$-manifold has a metric-compatible connection $\nabla^{-}$with holonomy contained in $\mathrm{SU}(3)$. If it coincides with the Levi-Cività connection $\nabla^{L C}$ one has a Calabi-Yau space, with $d \omega=d \Omega=0$. Furthermore, $\mathrm{SU}(3)$ structure manifolds are spin and carry a nontrivial, covariantly constant spinor (w.r.t. the canonical connection) of each chirality, $\eta$ and $\eta^{*}$, which are charge conjugates of each other. A nearly Kähler manifold is characterized by the conditions

$$
d \omega=-3 \varsigma \operatorname{Re}(\Omega), \quad d \Omega=2 i \varsigma \omega \wedge \omega \quad \text { with } \quad \varsigma \in \mathbb{R} .
$$

These forms can be constructed from the spinors $\eta$ and $\eta^{*}$ as

$$
\begin{aligned}
& \omega=\frac{i}{2} \bar{\eta} \gamma_{a} \gamma_{b} \eta e^{a} \wedge e^{b}, \\
& \Omega=+\frac{1}{6} \bar{\eta} \gamma_{a} \gamma_{b} \gamma_{c} \eta^{*} e^{a} \wedge e^{b} \wedge e^{c}, \\
& \bar{\Omega}=-\frac{1}{6} \bar{\eta}^{*} \gamma_{a} \gamma_{b} \gamma_{c} \eta e^{a} \wedge e^{b} \wedge e^{c},
\end{aligned}
$$

where the $e^{a}$ form an orthonormal frame of one-forms for $T^{*}(K)$. Every compact sixdimensional Einstein manifold carrying a nontrivial Killing spinor $\zeta$, i.e. one satisfying

$$
\nabla_{a} \zeta=\vartheta \gamma_{a} \zeta \quad \text { with } \quad \vartheta= \pm \frac{i}{2} \sqrt{\frac{\text { Scal }}{30}}
$$

has a nearly Kähler structure (the converse is also true [15]). Indeed, one can choose $\zeta$ to be of the form $\eta+\eta^{*}$ for some positive-chirality spinor $\eta$, which then defines the $\mathrm{SU}(3)$ structure $(J, \omega, \Omega)$ satisfying (3.1) with the particular value

$$
\varsigma=\sqrt{\frac{\text { Scal }}{30}} \quad \text { where } \quad \text { Scal }=\operatorname{Scal}\left(\nabla^{L C}\right) .
$$

Another important quantity in the study of a nearly Kähler manifold is its intrinsic torsion. This is defined as the torsion of the canonical connection, and it is totally antisymmetric, which is useful in regard of the supersymmetry equations. In this paper, we identify the intrinsic torsion with (one-half of) the $H$-flux

$$
H=\frac{1}{6} H_{a b c} e^{a} \wedge e^{b} \wedge e^{c} .
$$

In terms of the basic three-form, it is given by

$$
H=-\frac{i}{2} \varsigma(\Omega-\bar{\Omega})=\varsigma \operatorname{Im}(\Omega)
$$

Therefore, the canonical connection reads

$$
\nabla^{-}=\nabla^{L C}+\frac{1}{2} H_{b c}^{a} e^{b} \otimes\left(E_{a} \otimes e^{c}\right),
$$

with vector fields $E_{a}$ dual to the one-forms $e^{a}$. 
Properties of nearly Kähler manifolds. Let $K$ be a nearly Kähler manifold. The following properties of $K$ can all be proven by some elementary spinor calculus, the assumption that $\eta+\eta^{*}$ is a Killing spinor for the Levi-Cività connection on $K$, and multiple application of the following Fierz identities:

$$
\begin{aligned}
& \eta \bar{\eta}=\frac{1}{8}\left(1-\frac{1}{2}\left(\bar{\eta} \gamma_{a b} \eta\right) \gamma^{a b}\right)\left(1+(-1)^{\text {Chir }}\right), \\
& \eta^{*} \bar{\eta}=-\frac{1}{48} \Omega_{a b c} \gamma^{a} \gamma^{b} \gamma^{c} \\
& \eta \bar{\eta}^{*}=+\frac{1}{48} \bar{\Omega}_{a b c} \gamma^{a} \gamma^{b} \gamma^{c} .
\end{aligned}
$$

Here 'Chir' denotes the chirality operator, giving 0 on positive chirality spinors, and 1 on negative chirality. Our convention for the gamma-matrices is $\left\{\gamma^{a}, \gamma^{b}\right\}=2 g^{a b}$. The forms satisfy the duality relations

$$
* \Omega=-i \Omega, \quad * \bar{\Omega}=i \bar{\Omega}, \quad 2 * \omega=\omega \wedge \omega
$$

and they act on the spinors $\eta$ and $\eta^{*}$ as

$$
\begin{aligned}
\Omega_{a b c} \gamma^{c} \eta^{*} & =0, & \bar{\Omega}_{a b c} \gamma^{c} \eta & =0, \\
\Omega_{a b c} \gamma^{b} \gamma^{c} \eta & =-8 \gamma_{a} \eta^{*}, & \bar{\Omega}_{a b c} \gamma^{b} \gamma^{c} \eta^{*} & =8 \gamma_{a} \eta .
\end{aligned}
$$

Our forms are normalized as

$$
(\omega, \omega)=3, \quad(\Omega, \bar{\Omega})=8, \quad(\Omega, \Omega)=(\bar{\Omega}, \bar{\Omega})=0,
$$

where $(\cdot, \cdot)$ denotes the metric induced on $\Omega(K)$ by $g$. This implies

$$
\omega^{3}=6 \mathrm{Vol} \quad \text { and } \quad \Omega \wedge \bar{\Omega}=-8 i \mathrm{Vol}
$$

The derivatives $d \omega$ and $d \Omega$ are given in (3.1).

Coset models. There are four known compact six-dimensional nearly Kähler manifolds, which can all be represented as coset spaces $K=G / H$, for two Lie groups $H \subset G$, where $H$ is isomorphic to a subgroup of $\mathrm{SU}(3)$, and not to be confused with the torsion form $H \in \Omega(K)$ :

$$
\begin{array}{ll}
\mathrm{SU}(3) / \mathrm{U}(1) \times \mathrm{U}(1), & \mathrm{Sp}(2) / \mathrm{Sp}(1) \times \mathrm{U}(1), \\
G_{2} / \mathrm{SU}(3)=S^{6}, & \mathrm{SU}(2)^{3} / \mathrm{SU}(2)_{\operatorname{diag}}=S^{3} \times S^{3} .
\end{array}
$$

Their nearly Kähler structure comes from a so-called 3-symmetry, i.e. an automorphism

$$
s: G \rightarrow G \quad \text { with } \quad s^{3}=i d_{G} \text { and }\left.\quad s\right|_{H}=i d_{H} .
$$

For a precise definition consult [6]. The differential $d s: \mathfrak{g} \rightarrow \mathfrak{g}$ has three possible eigenvalues, namely

$$
1, \quad j=-\frac{1}{2}+i \frac{\sqrt{3}}{2}, \quad j^{2}=\bar{j}=-\frac{1}{2}-i \frac{\sqrt{3}}{2},
$$


with eigenspace decomposition $\mathfrak{g}^{\mathbb{C}}=\mathfrak{h}^{\mathbb{C}} \oplus \mathfrak{m}^{+} \oplus \mathfrak{m}^{-}$. Then $\mathfrak{m}=\left(\mathfrak{m}^{+} \oplus \mathfrak{m}^{-}\right) \cap \mathfrak{g}$ can be identified with the tangent space $T_{[e]} G / H$. Identifying $\mathfrak{m}^{+}$with $(1,0)$-type vectors and $\mathfrak{m}^{-}$ with (0,1)-type ones, we obtain an almost-complex structure $J$ on $G / H$, which relates to the 3 -symmetry via

$$
\left.d s\right|_{\mathfrak{m}}=-\frac{1}{2} i d+\frac{\sqrt{3}}{2} J
$$

This decomposition has the following properties,

$$
\begin{aligned}
{\left[\mathfrak{h}, \mathfrak{m}^{+}\right] } & \subset \mathfrak{m}^{+}, & {\left[\mathfrak{h}, \mathfrak{m}^{-}\right] } & \subset \mathfrak{m}^{-}, \\
{\left[\mathfrak{m}^{+}, \mathfrak{m}^{+}\right] } & \subset \mathfrak{m}^{-}, & {\left[\mathfrak{m}^{-}, \mathfrak{m}^{-}\right] } & \subset \mathfrak{m}^{+}, \\
{\left[\mathfrak{m}^{+}, \mathfrak{m}^{-}\right] } & \subset \mathfrak{h}^{\mathbb{C}}, & {[\mathfrak{h}, \mathfrak{h}] } & \subset \mathfrak{h} .
\end{aligned}
$$

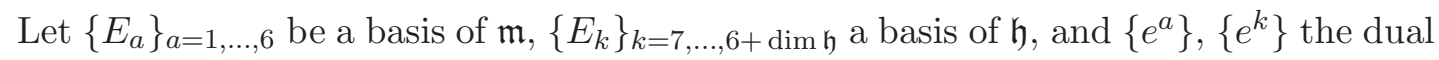
bases. As indicated, we use letters $a, b, c, \ldots$ for indices in $\mathfrak{m}$ and $i, j, k, \ldots$ for those in $\mathfrak{h}$. We also identify the $e^{a}$ and $e^{k}$ with the correponding left-invariant forms on $G$. Locally one can find a smooth map $\alpha: G / H \rightarrow G$, by which we pull back the forms on $G$ to forms on $G / H$, which we again denote by the same symbols. An important technical tool is the Maurer-Cartan equation

$$
\begin{aligned}
d e^{a} & =-\frac{1}{2} f_{b c}^{a} e^{b} \wedge e^{c}-f_{b k}^{a} e^{b} \wedge e^{k}, \\
d e^{k} & =-\frac{1}{2} f_{b c}^{k} e^{b} \wedge e^{c}-\frac{1}{2} f_{i j}^{k} e^{i} \wedge e^{j} .
\end{aligned}
$$

For the metric on $\mathfrak{m}$ we choose minus the Cartan-Killing form of $\mathfrak{g}$ restricted to $\mathfrak{m}$, i.e.

$$
g_{a b}=-f_{a d}^{c} f_{b c}^{d}-2 f_{a k}^{c} f_{b c}^{k} .
$$

Both terms on the right-hand side are separately proportional to the metric [16],

$$
f_{a d}^{c} f_{b c}^{d}=f_{a k}^{c} f_{b c}^{k}=-\frac{1}{3} g_{a b} .
$$

The last two equations fix the scale of the internal space $G / H$. This is done here for simplicity only; later on, we shall allow for a rescaling $\rho$ of the metric.

Connections and curvature in the coset models. Using the above mentioned properties, one can determine the Levi-Cività connection on $G / H$ by the conditions of metricity,

$$
0=E_{a} g_{b c}=\Gamma_{a b}^{d} g_{d c}+\Gamma_{a c}^{d} g_{b d}
$$

and the vanishing of the torsion,

$$
d e^{a}+\Gamma_{b}^{a} \wedge e^{b}=0
$$

One finds that

$$
\Gamma=\left(f_{i c}^{a} e^{i}+\frac{1}{2} f_{b c}^{a} e^{b}\right)\left(E_{a} \otimes e^{c}\right)
$$


We will need not only the Levi-Cività connection but a metric connection with torsion of the form

$$
T=\kappa f_{b c}^{a} e^{b} \otimes\left(E_{a} \otimes e^{c}\right) \in \Omega^{1}(\operatorname{End}(T K)) \quad \text { with } \quad \kappa \in \mathbb{R} .
$$

The canonical connection is included for $\kappa=-1 / 2$. Thus we have

$$
\Gamma^{\kappa}=\left(f_{i c}^{a} e^{i}+\frac{1}{2} \phi f_{b c}^{a} e^{b}\right)\left(E_{a} \otimes e^{c}\right) \quad \text { with } \quad \phi:=2 \kappa+1 .
$$

The curvature tensors of $\Gamma^{\kappa}$ are found to be

$$
\begin{aligned}
R^{\kappa} & =-\frac{1}{4}\left\{\phi f_{a b}^{e} f_{e d}^{c}+2 f_{a b}^{k} f_{k d}^{c}-\phi^{2} f_{a e}^{c} f_{b d}^{e}\right\} e^{a} \wedge e^{b}\left(E_{c} \otimes e^{d}\right), \\
\operatorname{Ric}^{\kappa} & =-\frac{1}{12}\left(4 \kappa^{2}-5\right) g, \\
\mathrm{Scal}^{\kappa} & =-\frac{1}{2} \phi^{2}+\phi+2=-2 \kappa^{2}+\frac{5}{2} .
\end{aligned}
$$

In the following we will denote $\nabla^{\kappa}$ for special values of $\kappa$ as

$$
\begin{aligned}
& \nabla^{-\frac{1}{2}}=: \nabla^{-} \quad \text { canonical connection } \quad \Longrightarrow \quad R^{-\frac{1}{2}}=: R^{-} \text {, } \\
& \nabla^{0}=: \nabla \quad \text { Levi-Cività connection } \quad \Longrightarrow \quad R^{0}=: R \text {, } \\
& \nabla^{+\frac{1}{2}}=: \nabla^{+} \quad \text { no name } \quad \Longrightarrow \quad R^{+\frac{1}{2}}=: R^{+} \text {, }
\end{aligned}
$$

The curvatures $R^{ \pm}$are sometimes denoted as $\left.R^{ \pm}\right|_{\mathfrak{m}}$, in order to distinguish them from the curvature $\left.R^{-}\right|_{\mathfrak{h}}$ of another so-called canonical connection on the principal $H$-bundle $G \rightarrow G / H$. The latter acts in the adjoint representation on $\mathfrak{h}$, but it has the same functional form as $\left.R^{-}\right|_{\mathfrak{m}}[16,19]$. Explicit formulae are

$$
\begin{aligned}
\left.R^{+}\right|_{\mathfrak{m}} & =-\operatorname{ad}\left(E_{a}\right) \circ \pi_{\mathfrak{h}} \circ \operatorname{ad}\left(E_{b}\right) e^{a} \wedge e^{b}, & \left(R^{+}\right)^{c}{ }_{d a b} & =2 f_{k[a}^{c} f_{b] d}^{k}, \\
\left.R^{-}\right|_{\mathfrak{m}} & =-\frac{1}{2} f_{a b}^{k} \operatorname{ad}_{\mathfrak{m}}\left(E_{k}\right) e^{a} \wedge e^{b}, & \left(\left.R^{-}\right|_{\mathfrak{m}}\right)^{c}{ }_{d a b} & =-f_{a b}^{k} f_{k d}^{c}, \\
\left.R^{-}\right|_{\mathfrak{h}} & =-\frac{1}{2} f_{a b}^{k} \operatorname{ad}_{\mathfrak{h}}\left(E_{k}\right) e^{a} \wedge e^{b}, & \left(\left.R^{-}\right|_{\mathfrak{h}}\right)^{k}{ }_{l a b} & =-f_{a b}^{m} f_{m l}^{k},
\end{aligned}
$$

where $\pi_{\mathfrak{h}}$ is the projection of $\mathfrak{g}$ onto $\mathfrak{h}$. The torsion three-form of the canonical connection $\nabla^{-}$reads

$$
H=-\frac{1}{6} f_{a b c} e^{a} \wedge e^{b} \wedge e^{c} .
$$

From the explicit expression for $R^{\kappa}$ above we compute various curvature bilinears for later use:

$$
\begin{aligned}
\operatorname{tr}_{\mathfrak{m}} R^{+} \wedge R^{+} & =-\frac{1}{4}\left\langle E_{k}, E_{l}\right\rangle_{\mathfrak{h}} f_{a b}^{k} f_{c d}^{l} e^{a b c d} \\
\operatorname{tr}_{\mathfrak{m}} R^{-} \wedge R^{-} & =+\frac{1}{4}\left\langle E_{k}, E_{l}\right\rangle_{\mathfrak{m}} f_{a b}^{k} f_{c d}^{l} e^{a b c d} \\
\operatorname{tr}_{\mathfrak{h}} R^{-} \wedge R^{-} & =+\frac{1}{4}\left\langle E_{k}, E_{l}\right\rangle_{\mathfrak{h}} f_{a b}^{k} f_{c d}^{l} e^{a b c d} \\
d H & =-\frac{1}{4} f_{a b k} f_{c d}^{k} e^{a b c d}
\end{aligned}
$$


where tr denotes minus the usual trace, $e^{a b c d}=e^{a} \wedge e^{b} \wedge e^{c} \wedge e^{d}$, and

$$
\left\langle E_{k}, E_{l}\right\rangle_{\mathfrak{h}}=-f_{k n}^{m} f_{l m}^{n} \quad \text { as well as } \quad\left\langle E_{k}, E_{l}\right\rangle_{\mathfrak{m}}=-f_{k b}^{a} f_{l a}^{b}
$$

are minus the Cartan-Killing forms on $\mathfrak{h}$ and $\mathfrak{m}$, respectively. On three of the four nearly Kähler cosets $G / H$ (those where $\mathfrak{h}$ is semisimple) we have the identity

$$
\langle\cdot, \cdot\rangle_{\mathfrak{h}}=\beta\langle\cdot, \cdot\rangle_{\mathfrak{g}}
$$

for a specific value of $\beta$. The value of $\beta$ can be calculated in orthonormal coordinates on $G$ as follows:

$$
f_{a b}^{k} f_{a b}^{k}=\frac{1}{3} \delta_{a a}=2 \quad \text { and } \quad f_{a b}^{k} f_{a b}^{k}=(1-\beta) \delta^{k k}=(1-\beta) \operatorname{dim}(H) \quad \Longrightarrow \quad \beta=1-\frac{2}{\operatorname{dim}(H)},
$$

for semisimple $\mathfrak{h}$. For our examples this yields

\begin{tabular}{|c|c|c|c|c|}
\hline & $\mathrm{SU}(3) / \mathrm{U}(1) \times \mathrm{U}(1)$ & $\mathrm{Sp}(2) / \mathrm{Sp}(1) \times \mathrm{U}(1)$ & $G_{2} / \mathrm{SU}(3)$ & $\mathrm{SU}(2)^{3} / \mathrm{SU}(2)$ \\
\hline$\beta$ & 0 & - & $3 / 4$ & $1 / 3$ \\
\hline
\end{tabular}

and we get $(\phi=2 \kappa+1)$

$$
\begin{aligned}
\operatorname{tr}_{\mathfrak{m}} R^{+} \wedge R^{+} & =\beta d H, \\
\operatorname{tr}_{\mathfrak{m}} R^{-} \wedge R^{-} & =(\beta-1) d H, \\
\operatorname{tr}_{\mathfrak{h}} R^{-} \wedge R^{-} & =-\beta d H, \\
\operatorname{tr}_{\mathfrak{m}} R^{\kappa} \wedge R^{\kappa} & =\left(\beta+\frac{1}{4} \phi^{2}-1\right) d H, \\
\operatorname{tr}_{\mathfrak{m}}\left|R^{+}\right|^{2} & =\frac{4}{3}-\beta, \\
\operatorname{tr}_{\mathfrak{m}}\left|R^{-}\right|^{2} & =1-\beta, \\
\operatorname{tr}_{\mathfrak{h}}\left|R^{-}\right|^{2} & =\beta \\
\operatorname{tr}_{\mathfrak{m}}\left|R^{\kappa}\right|^{2} & =\frac{1}{24} \phi^{2}\left(\phi^{2}-2\right)+1-\beta, \\
R_{\text {acde }}^{+} R_{b}^{+c d e} & =\frac{4-3 \beta}{9} g_{a b}, \\
R_{\text {acde }}^{-} R_{b}^{-c d e} & =\frac{1-\beta}{3} g_{a b} .
\end{aligned}
$$

In the compactifications we consider, the curvature $\tilde{R}$ of the tangent bundle of $K$ is one of the $R^{\kappa}$, usually either $R^{+}$or $R^{-}$. In contrast, the gauge field $F$ is free to live on an arbitrary bundle, and so we also have the choice $F=\left.R^{-}\right|_{\mathfrak{h}}$ at our disposal. The supersymmetry constraint, however, forces $F$ to be a (generalized) instanton, meaning that $* F=-\omega \wedge F$. This is satisfied only by $R^{-}$(both on $\mathfrak{m}$ or on $\mathfrak{h}$ ). If $\mathfrak{h}$ is abelian, we will also have the freedom to rescale $\Gamma^{-}$without losing the instanton property. Hence, for $\mathrm{SU}(3) / \mathrm{U}(1) \times \mathrm{U}(1)$ we may take $F=\lambda R^{-}$with $\lambda \in \mathbb{R}$ (cf. [23]). Yet even without the supersymmetry constraint it is very convenient to choose an instanton solution for the gauge field because it automatically satisfies the Yang-Mills equation. 
On the space $\operatorname{Sp}(2) / \operatorname{Sp}(1) \times \mathrm{U}(1)$ there is no common value for $\beta$. Instead, we have $\beta=0$ on $\mathfrak{u}(1)$ and $\beta=2 / 3$ on $\mathfrak{s p}(1)$. This allows one to calculate the quantities $\operatorname{tr}\left(R^{ \pm} \wedge R^{ \pm}\right)$, which are no longer proportional to $d H$. However, we have the freedom to restrict the curvature $\tilde{R}$ to the $\mathfrak{u}(1)$ part of $\mathfrak{h}$. Again this can be rescaled, enabling us to satisfy the Bianchi identity for a particular choice,

$\tilde{R}=\left.R\right|_{\mathfrak{u}(1)} \quad$ and $\quad F=\left.R^{-}\right|_{\mathfrak{m}} \quad \Longrightarrow \quad \operatorname{tr}(\tilde{R} \wedge \tilde{R}-F \wedge F)=r d H \quad$ with $\quad r=1 / 3$.

In contrast to the $\mathrm{SU}(3) / \mathrm{U}(1) \times \mathrm{U}(1)$ case, there are then no free parameters left in the gauge field. We will find below that only $r=2 / 3$ and $r=4 / 9$ are compatible with the dilaton equation, in the supersymmetric and non-supersymmetric case, respectively. Therefore we will not consider this coset space any further.

Forms in the coset models. Now we can compare the general theory to our concrete realization in terms of coset models. In particular, we identified

$$
T_{a b}^{c}=-\frac{1}{2} f_{a b}^{c} \quad \text { and } \quad \mathrm{Scal} \equiv \mathrm{Scal}^{0}=\frac{5}{2} \quad \Longrightarrow \quad \varsigma=\sqrt{\frac{\mathrm{Scal}}{30}}=\sqrt{\frac{1}{12}} .
$$

From this we easily deduce

$$
(\Omega-\bar{\Omega})_{a b c}=-4 \sqrt{3} i f_{a b c} \quad \text { and } \quad H_{a b c}=-f_{a b c}
$$

as well as the relations

$$
\begin{aligned}
& d \omega=-\frac{3}{2} \varsigma(\Omega+\bar{\Omega})=-\frac{\sqrt{3}}{4}(\Omega+\bar{\Omega})=3 * H, \\
& d \Omega=-d \bar{\Omega}=2 i \varsigma \omega \wedge \omega=\frac{1}{\sqrt{3}} i \omega \wedge \omega, \\
& d H=\frac{1}{15} \operatorname{Scal} \omega \wedge \omega=\frac{1}{6} \omega \wedge \omega .
\end{aligned}
$$

With $\varsigma$ we have fixed the scale of the nearly Kähler manifold. Let us finally see what happens if we relax this scale by allowing for an arbitrary value of $\varsigma$. The new metric is then $g^{\prime}=\rho g$, with $\rho=\frac{1}{12 \varsigma^{2}}$. Denoting $g^{\prime}$ by $g$ again, we get the relations

$$
\begin{aligned}
\text { Ric } & =\frac{5}{12} \rho^{-1} g, & \text { Scal } & =\frac{5}{2} \rho^{-1}, \\
|H|^{2} & =\frac{1}{3} \rho^{-1}, & H_{a c d} H_{b}{ }^{c d} & =\frac{1}{3} \rho^{-1} g_{a b},
\end{aligned}
$$

and the quantities given in (3.34) scale as follows,

$$
\operatorname{tr} R \wedge R \sim \rho^{-1} d H, \quad \operatorname{tr}|R|^{2} \sim \rho^{-2}, \quad R_{a c d e} R_{b}{ }^{c d e} \sim \rho^{-2} g_{a b} .
$$

\section{Supersymmetric solutions with gaugino condensate}

In $[10,13]$ solutions of the supersymmetry equations (2.8) were constructed on the product of $\mathrm{AdS}_{4}$ with a nearly Kähler space $K$, by setting $\phi=0$ and $H=-\frac{1}{4} \Sigma$ equal to the 
intrinsic torsion of $K$. The metric on $K=G / H$ is a scale factor $\rho$, to be determined below, multiplied by (the negative of) the Killing form of $G$. Frey and Lippert in [10] also propose a certain ansatz for the gauge field, which is not an instanton however and therefore has a non-vanishing gaugino variation. We will discuss the choice of the gauge field later, together with the Bianchi identity and dilaton equation. The supersymmetry generator $\varepsilon$ is obtained as follows [10]: $\operatorname{AdS}_{4}(r)$ carries a Killing spinor $\widehat{\zeta}+\widehat{\zeta}^{*}$ with Killing number $\vartheta=\frac{1}{2 r}=\frac{1}{\sqrt{2 \alpha^{\prime}}}[2,5]$, i.e.

$$
\nabla_{\mu} \widehat{\zeta}=\vartheta \gamma_{\mu} \widehat{\zeta}^{*} \quad \text { and } \quad \nabla_{\mu} \widehat{\zeta}^{*}=\vartheta \gamma_{\mu} \widehat{\zeta}
$$

On $K$ we have the Killing spinor $\eta$ (of positive chirality) with $\nabla^{-} \eta=\nabla^{-} \eta^{*}=0$, which gives

$$
\varepsilon=e^{\frac{i \pi}{4}} \widehat{\zeta} \otimes \eta+e^{-\frac{i \pi}{4} \widehat{\zeta}^{*}} \otimes \eta^{*} .
$$

Apparently the dilatino variation $\delta \lambda$ in (2.8) vanishes, and for the $\mathrm{AdS}_{4}$ components of $\delta \psi_{\mu}$ we obtain

$$
\begin{aligned}
\delta \psi_{\mu} & =\nabla_{\mu} \varepsilon+\frac{1}{96} \gamma(\Sigma) \gamma_{\mu} \varepsilon \\
& =e^{\frac{i \pi}{4}} \vartheta \gamma_{\mu} \widehat{\zeta}^{*} \otimes \eta+e^{-\frac{i \pi}{4}} \vartheta \gamma_{\mu} \widehat{\zeta} \otimes \eta^{*}-\frac{1}{96} \gamma_{\mu} \gamma(\Sigma)\left(e^{\frac{i \pi}{4}} \widehat{\zeta} \otimes \eta+e^{-\frac{i \pi}{4}} \widehat{\zeta}^{*} \otimes \eta^{*}\right) .
\end{aligned}
$$

The condition $\Sigma=-4 H$ fixes the condensate scale $\Lambda$ in the condensate (2.24). Together with (3.6), it follows that

$$
\Sigma=2 i \varsigma(\Omega-\bar{\Omega}) \quad \text { and } \quad \varsigma=\sqrt{\frac{\text { Scal }}{30}}=\sqrt{\frac{\rho}{12}} \quad \Leftrightarrow \quad \rho=\frac{1}{12 \varsigma^{2}},
$$

relating $\varsigma$ to the scale $\rho$ of $K$. Together with (3.10) this implies

$$
\gamma(\Sigma) \eta=-96 i \varsigma \eta^{*} \quad \text { and } \quad \gamma(\Sigma) \eta^{*}=-96 i \varsigma \eta .
$$

Finally we get

$$
\delta \psi_{\mu}=(\vartheta-\varsigma)\left[e^{\frac{i \pi}{4}} \gamma_{\mu} \widehat{\zeta}^{*} \otimes \eta+e^{-\frac{i \pi}{4}} \gamma_{\mu} \widehat{\zeta} \otimes \eta^{*}\right] \quad \Longrightarrow \quad \vartheta=\varsigma .
$$

The $K$ component $\delta \psi_{a}$ gives zero as well, due to $\gamma(\Sigma) \gamma_{a} \eta=\gamma(\Sigma) \gamma_{a} \eta^{*}=0$.

Implications of supersymmetry. Due to the condition $\vartheta=\varsigma$ we can determine the total scalar curvature of our space $\mathrm{AdS}_{4} \times K$ :

$$
\widehat{\mathrm{Scal}}+\mathrm{Scal}=-48 \vartheta^{2}+30 \varsigma^{2}=-18 \vartheta^{2}=-\frac{9}{\alpha^{\prime}},
$$

which is strictly negative. Furthermore,

$$
\Sigma=-4 H \quad \Longrightarrow \quad T=H-\frac{1}{2} \Sigma=3 H,
$$

which turns the gaugino equation (left of (2.22)) into the form

$$
\left(\mathcal{D}-\frac{1}{8} \gamma(H)\right) \chi=0 .
$$

This will be satisfied if we decompose the gaugino as in (2.20), with $\eta$ being the Killing spinor of $K$ [10]. Having adjusted the value of $\Lambda$, the three-form $\Sigma$ assumes the required structure $\Sigma=2 i \varsigma(\Omega-\bar{\Omega})$. 
Einstein and dilaton equations. The external Einstein equation (the first one in (2.25)) was analyzed in section 2 , yielding

$$
r^{2}=\frac{1}{4 \vartheta^{2}}=\frac{\alpha^{\prime}}{2} \quad \Longrightarrow \quad \varsigma^{2}=\frac{1}{2 \alpha^{\prime}} \quad \text { and } \quad \rho=\frac{\alpha^{\prime}}{6} .
$$

Next, we want to consider the fourth equation in (2.25), which can be written as

$$
\widehat{\mathrm{Scal}}+\mathrm{Scal}+\frac{9}{2}|H|^{2}=0 \text {. }
$$

The normalization of the intrinsic torsion $H$ was such that $|H|^{2}=4 \varsigma^{2}$, and together with (4.7) and (4.6), we deduce that (4.11) is satisfied.

Let us then determine the remaining conditions for $\tilde{R}$ and $F$ following from the equations of motion. Due to (4.8), the third equation in (2.25) becomes

$$
\frac{1}{4} \alpha^{\prime}\left[\frac{12}{r^{4}}+\operatorname{tr}\left(|\tilde{R}|^{2}-|F|^{2}\right)\right]=3|H|^{2}=12 \varsigma^{2},
$$

and the Bianchi identity remains unchanged:

$$
d H=\frac{1}{4} \alpha^{\prime} \operatorname{tr}[\tilde{R} \wedge \tilde{R}-F \wedge F] .
$$

Written in terms of $\rho=\frac{1}{12 \varsigma^{2}}=\frac{\alpha^{\prime}}{6}$, we arrive at the conditions

$$
\operatorname{tr}\left[|\tilde{R}|^{2}-|F|^{2}\right]=-\frac{2}{3} \rho^{-2} \quad \text { and } \quad \operatorname{tr}[\tilde{R} \wedge \tilde{R}-F \wedge F]=\frac{2}{3} \rho^{-1} d H .
$$

Further we need the Einstein equation on $K$ (the second one in (2.25)), which must imply (4.12). It is easy to see that all terms in this equation are proportional to $g_{a b}$, so that it becomes equivalent to its trace (4.12).

Choice of connections. The gaugino equation (left of (2.22)) is satisfied because $\eta$ is parallel with respect to $\nabla^{-}$. The Kalb-Ramond equation is solved due to (3.1), (3.6) and (3.9). The Yang-Mills equation will be obeyed for (generalized) instanton connections. The nearly Kähler geometry of $K$ determines $H$, but for $\tilde{R}$ and $F$ different choices are possible, the consequences of which can be computed using (3.34). To this end, the following lemma will be useful:

Lemma 4.1. Let $F$ be a (generalized) instanton, i.e. $F$ satisfies the $\omega$-anti-self-duality relation

$$
* F=-\omega \wedge F,
$$

(which is equivalent to the vanishing of the gaugino supersymmetry variation). Suppose further that

$$
\operatorname{tr}(F \wedge F)=\frac{\varkappa}{\rho} d H
$$

Then it follows that

$$
\operatorname{tr}|F|^{2}=-\frac{\varkappa}{\rho^{2}} .
$$


Proof. We have

$$
\operatorname{tr}|F|^{2}=\operatorname{tr} *(F \wedge * F)=-\operatorname{tr} *(F \wedge \omega \wedge F)=-\frac{\varkappa}{\rho} *(\omega \wedge d H)=-\frac{\varkappa}{6 \rho^{2}} * \omega^{3}=-\frac{\varkappa}{\rho^{2}},
$$

where the relations $\omega^{3}=6 \mathrm{Vol}$ and $d H=\frac{1}{6 \rho} \omega \wedge \omega$ for nearly Kähler spaces in our particular normalization have been used.

The lemma implies that if both $\tilde{R}$ and $F$ are instantons, with $\operatorname{tr}(F \wedge F) \sim d H$, then the two equations (4.14) become equivalent to one another, and we need to solve only one of them. Hence, in this case the vanishing of the supersymmetry constraints plus the Bianchi identity imply the field equations, as expected.

The gauge field $A$ proposed by Frey and Lippert is the torsionful connection $\Gamma^{\kappa}$ as in (3.25), where the value of $\kappa$ has to be adjusted to the Bianchi identity. However, they employ $\tilde{\Gamma}=\Gamma^{+}{ }^{5}$ The problem with this choice is that $\Gamma^{\kappa}$ is in general not an instanton, leading to a non-vanishing gaugino variation. We will therefore consider $\tilde{\Gamma}=\Gamma^{-}$and other possibilities of the gauge connection as well.

The minus-connection: $\tilde{\boldsymbol{\Gamma}}=\boldsymbol{\Gamma}^{-}$. First we consider solutions where $\tilde{R}=\left.R^{-}\right|_{\mathfrak{m}}$ and $F$ is also an instanton, i.e. $F=\left.R^{-}\right|_{\mathfrak{h}}$ or, for $\mathfrak{h}$ abelian, $F=\left.\lambda R^{-}\right|_{\mathfrak{m}}$ with $\lambda \in \mathbb{R}$. The Bianchi identity reads

$$
\operatorname{tr} F \wedge F=\left(\beta-\frac{5}{3}\right) \rho^{-1} d H
$$

Choosing the canonical $H$-connection

$$
F=\left.R^{-}\right|_{\mathfrak{h}} \quad \text { with } \quad \operatorname{tr}(F \wedge F)=-\frac{\beta}{\rho} d H \quad \Longrightarrow \quad \beta=\frac{5}{6},
$$

which is not among the admissible values. A solution is obtained however on $\mathrm{SU}(3) / \mathrm{U}(1) \times$ $\mathrm{U}(1)(\beta=0)$, where we can choose

$$
F=\left.\sqrt{\frac{5}{3}} R^{-}\right|_{\mathfrak{m}} \quad \Longrightarrow \quad \operatorname{tr}(F \wedge F)=-\frac{5}{3} d H .
$$

The explicit expressions for $F$ and $\tilde{R}$ can be found in (3.28).

The plus-connection: $\tilde{\boldsymbol{\Gamma}}=\Gamma^{+}$. Now we turn to $\tilde{R}=R^{+}$solutions, so that $\tilde{R}$ is not an instanton any more. Here, $\operatorname{tr}\left(R^{+} \wedge R^{+}\right)$and $\operatorname{tr}\left|R^{+}\right|^{2}$ have been calculated in (3.34) and lead to the conditions

$$
\begin{aligned}
& \operatorname{tr} F \wedge F=\left(\beta-\frac{2}{3}\right) \rho^{-1} d H \quad \text { (Bianchi), } \\
& \operatorname{tr}|F|^{2}=(2-\beta) \rho^{-2} \quad \text { (Dilaton), }
\end{aligned}
$$

in contradiction with Lemma 4.1. The Bianchi identity on $\mathrm{SU}(2)^{3} / \mathrm{SU}(2)$ with $\beta=1 / 3$ can be obeyed for $F=\left.R^{-}\right|_{\mathfrak{h}}$, but this choice fails to solve the dilaton equation (second of (2.13)). Thus we confirm Ivanov's result that only for $\tilde{\Gamma}=\Gamma^{-}$the field equations

\footnotetext{
${ }^{5}$ Note that our $\Gamma^{+}$is their $\Gamma^{-}$.
} 
follow. The string theory formulated in this background then has a conformal anomaly, but is otherwise consistent [22]. The situation can be remedied by turning on a dilatino condensate. This introduces the freedom necessary to generate consistent solutions with $\tilde{\Gamma}=\Gamma^{+}$on all nearly Kähler spaces [21].

\section{Non-supersymmetric solutions with vanishing gaugino}

We will now construct a solution of the bosonic equations of motion on $\mathrm{AdS}_{4} \times K$ with vanishing gaugino, which necessarily breaks supersymmetry. As before we choose the $H$ field equal to the intrinsic torsion.

Equations of motion for $\chi=\mathbf{0}$. For $\phi=0$ and $\chi=0$ the equations (2.13) simplify to

$$
\begin{aligned}
& |H|^{2}=\frac{1}{4} \alpha^{\prime} \operatorname{tr}\left[\frac{12}{r^{4}}+|\tilde{R}|^{2}-|F|^{2}\right] \quad \Longrightarrow \quad \frac{48}{{\alpha^{\prime}}^{2}}=\frac{4}{\alpha^{\prime}}|H|^{2}+\operatorname{tr}|F|^{2}-\operatorname{tr}|\tilde{R}|^{2}, \\
& 0=-\frac{24}{r^{2}}+2 \mathrm{Scal}+|H|^{2} \quad \Longrightarrow \quad \frac{24}{\alpha^{\prime}}=\mathrm{Scal}+\frac{1}{2}|H|^{2} \quad \Longrightarrow \quad \rho=\frac{\alpha^{\prime}}{9},
\end{aligned}
$$

by virtue of $r^{2}=\frac{\alpha^{\prime}}{2}$. The value for $\rho$ differs from the supersymmetric one (4.7). The Bianchi identity and the remaining equations of motion become

$$
\begin{aligned}
d H & =\frac{1}{4} \alpha^{\prime} \operatorname{tr}[\tilde{R} \wedge \tilde{R}-F \wedge F], \\
0 & =d * H, \\
0 & =d F+A \wedge * F-* F \wedge A+* H \wedge F, \\
0 & =\operatorname{Ric}_{a b}-\frac{1}{4} H_{a c d} H_{b}{ }^{c d}+\frac{1}{4} \alpha^{\prime}\left[\tilde{R}_{a c d e} \tilde{R}_{b}^{c d e}-\operatorname{tr} F_{a c} F_{b}^{c}\right] \\
& =\frac{1}{3} g_{a b}+\frac{1}{4} \alpha^{\prime}\left[\tilde{R}_{a c d e} \tilde{R}_{b}^{c d e}-\operatorname{tr} F_{a c} F_{b}^{c}\right],
\end{aligned}
$$

Since all terms in the Einstein equation are proportional to $g_{a b}$, its information is already contained in the trace,

$$
\frac{36}{\left(\alpha^{\prime}\right)^{2}}=\operatorname{tr}\left[|F|^{2}-|\tilde{R}|^{2}\right]
$$

which is just the first equation of (5.1). We will solve the Yang-Mills equation simply by imposing the instanton condition on $F$, so that all equations are satisfied, except for the Bianchi identity and the dilaton equation (5.3). Let us again assume that both $F$ and $\tilde{R}$ satisfy the instanton condition, as well as

$$
\operatorname{tr}(\tilde{R} \wedge R)=\frac{\varkappa_{1}}{\rho} d H \quad \text { and } \quad \operatorname{tr}(F \wedge F)=\frac{\varkappa_{2}}{\rho} d H .
$$

Using lemma 4.1, we can rewrite these two conditions as

$$
\left(\frac{\varkappa_{1}}{\rho}-\frac{\varkappa_{2}}{\rho}\right)=\frac{4}{\alpha^{\prime}} \quad \text { and } \quad\left(\frac{\varkappa_{1}}{\rho^{2}}-\frac{\varkappa_{2}}{\rho^{2}}\right)=\frac{36}{\left(\alpha^{\prime}\right)^{2}} .
$$


The minus-connection: $\tilde{\boldsymbol{\Gamma}}=\boldsymbol{\Gamma}^{-}$. Let us try to fulfil the equations using $\Gamma^{-}$. According to the discussion above, a single equation remains to be solved:

$$
\operatorname{tr} F \wedge F=\left(\beta-\frac{13}{9}\right) \rho^{-1} d H
$$

The most obvious choice is the canonical $H$-connection, i.e. $\left.\Gamma^{-}\right|_{\mathfrak{h}}$. This however has $\operatorname{tr}_{\mathfrak{h}} F \wedge F=-\beta d H$, so that (5.6) implies $\beta=\frac{13}{18}$, which is not admissible. Only on $\mathrm{SU}(3) / \mathrm{U}(1) \times \mathrm{U}(1)$ we achieve a solution for $F=\left.\sqrt{\frac{13}{9}} R^{-}\right|_{\mathfrak{m}}$.

The plus-connection: $\tilde{\boldsymbol{\Gamma}}=\boldsymbol{\Gamma}^{+}$. In this case, the Bianchi identity and Einstein equation read

$$
\operatorname{tr} F \wedge F=\left(\beta-\frac{4}{9}\right) \rho^{-1} d H \quad \text { and } \quad \operatorname{tr}|F|^{2}=\frac{16-9 \beta}{9 \rho^{2}} .
$$

These equations do not admit instanton solutions for $F$, as

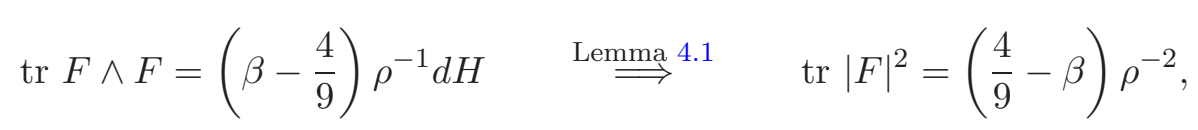

which leads to the contradiction $\frac{4}{9}-\beta=\frac{16}{9}-\beta$.

Furthermore, there are no models with a conformal anomaly, satisfying only the Bianchi identity. Non-instanton solutions are not excluded by this argument, but these have the drawback that the Yang-Mills equations have to be checked explicitly.

Another possibility is to allow for more general torsionful connections, $\tilde{\Gamma}=\Gamma^{\kappa}$, and maybe also $F=\Gamma^{\kappa^{\prime}}$. This does not lead to further solutions however, as one can see from the relevant expressions calculated in (3.34).

The volume modulus. One may try to deform our supersymmetric solution to obtain a family of solutions, possibly relaxing the supersymmetry constraint. A simple option is a rescaling

$$
\chi^{\prime}=\tau \chi, \quad g^{\prime}=\rho g \quad \text { and } \quad F^{\prime}=\theta F .
$$

In this case, the dilaton and Einstein equations together with the Bianchi identity imply

$$
\tau=0 \quad \text { or } \quad \tau=1 \quad \Longrightarrow \quad \alpha^{\prime}=9 \rho \quad \text { or } \quad \alpha^{\prime}=6 \rho
$$

and the corresponding values for $F$ we found above. In particular, our two solutions to the equations of motion cannot be continuously connected by an obvious path in the field space.

\section{Conclusions}

We found that for two nearly Kähler spaces, $\mathrm{SU}(2)^{3} / \mathrm{SU}(2)$ and $\mathrm{SU}(3) / \mathrm{U}(1) \times \mathrm{U}(1)$, the supersymmetric model of Frey and Lippert can be completed by an appropriate choice of gauge field, and that the equations of motion are satisfied on the second space, whereas the first one has a conformal anomaly at order $\alpha^{\prime}$, i.e. the dilaton equation (fourth of (2.23)) does not hold. This is due to the choice $\tilde{\Gamma}=\Gamma^{+}$required by the $\mathrm{SU}(2)^{3} / \mathrm{SU}(2)$-model, 
whereas on $\mathrm{SU}(3) / \mathrm{U}(1) \times \mathrm{U}(1)$ we can work with the instanton connection $\tilde{\Gamma}=\Gamma^{-}$. The explicit form of our solution on $\mathrm{AdS}_{4}(r) \times \mathrm{SU}(3) / \mathrm{U}(1) \times \mathrm{U}(1)$ with radius $r=\sqrt{\alpha^{\prime} / 2}$ and internal scale $\rho=\alpha^{\prime} / 6$ reads

$$
\begin{aligned}
g_{a b} & =-\frac{1}{2} \alpha^{\prime} f_{a d}^{c} f_{b c}^{d}=-\frac{1}{2} \alpha^{\prime} f_{a k}^{c} f_{b c}^{k}, \\
(\tilde{\Gamma})_{a}^{c} & =\left(\Gamma^{-}\right)_{a}^{c}=f_{i c}^{a} e^{i}, \\
T_{a b c} & =3 H_{a b c}=-3 f_{a b c}, \\
F_{a b} & =\left.\sqrt{\frac{5}{3}} R_{a b}^{-}\right|_{\mathfrak{m}}=-\sqrt{\frac{5}{3}} f_{a b}^{k} \operatorname{ad}\left(E_{k}\right), \\
\phi & =\psi=\lambda=0, \\
\chi & =\widehat{\chi} \otimes \eta+\widehat{\chi}^{*} \otimes \eta^{*} \quad \text { and } \quad\left\langle\Sigma_{a b c}\right\rangle=-\frac{4}{3} T_{a b c}=4 f_{a b c} .
\end{aligned}
$$

On the same space (but with $\rho=\alpha^{\prime} / 9$ ) we were also able to construct a solution to the equations of motion with non-vanishing fermionic supersymmetry variations,

$$
\begin{aligned}
g_{a b} & =-\frac{1}{3} \alpha^{\prime} f_{a d}^{c} f_{b c}^{d}=-\frac{1}{3} \alpha^{\prime} f_{a k}^{c} f_{b c}^{k}, \\
(\tilde{\Gamma})_{a}^{c} & =\left(\Gamma^{-}\right)_{a}^{c}=f_{i c}^{a} e^{i}, \\
T_{a b c} & =H_{a b c}=-f_{a b c}, \\
F_{a b} & =\left.\sqrt{\frac{13}{9}} R_{a b}^{-}\right|_{\mathfrak{m}}=-\sqrt{\frac{13}{9}} f_{a b}^{k} \operatorname{ad}\left(E_{k}\right), \\
\phi & =\psi=\lambda=\chi=0 \quad \Longrightarrow \quad\langle\Sigma\rangle=0 .
\end{aligned}
$$

It was known previously [21] that there exist complete solutions of the supersymmetry constraints and Bianchi identity (with $\tilde{\Gamma}=\Gamma^{+}$) on every nearly Kähler manifold if one includes a dilatino condensate, but the derivation of the equations of motion for this case is more involved. In the limit of vanishing dilatino condensate, these solutions still differ from ours.

A nice feature of the space-time Einstein equation with $\alpha^{\prime}$ correction,

$$
\operatorname{Ric}_{\mu \nu}+\frac{1}{4} \alpha^{\prime} R_{\mu \alpha \beta \gamma} R_{\nu}^{\alpha \beta \gamma}=0,
$$

is that it fixes the radius of $\mathrm{AdS}_{4}$ (in terms of $\alpha^{\prime}$ ) and thereby, in combination with the vanishing of the gravitino variation, also the scale of the internal manifold. There is thus no volume modulus in the game, an argument which apparently does not apply to Minkowski compactifications. It is not the supersymmetry constraints which fix the $\mathrm{AdS}_{4}$ radius; even our non-supersymmetric solution seems to have no volume modulus.

The length scale $r$ of both the internal and external manifold was found to be of the order of the string length $\sqrt{\alpha^{\prime}}$. To arrive at the solutions we truncated the equations of motion, supersymmetry transformations, and Bianchi identity at order $\left(\alpha^{\prime}\right)^{1}$, assuming higher order terms to be small. These equations are really expansions in $\alpha^{\prime} / r^{2}$ however, which is of order one and thus not a good expansion parameter. It is therefore unclear 
whether the solutions extend to full non-perturbative ones, and even if they do one has to expect large corrections to the relation between $r$ and $\alpha^{\prime}$ we found.

To address this problem one could try to study the world-sheet string theory in a background of the form $\mathrm{AdS}_{4}$ times nearly Kähler. Another problem that might find its solution in such an approach is the determination of the effective 4D superpotential. In [10] it was shown that the perturbative superpotential is independent of the gaugino condensate, whereas non-perturbative contributions are expected to generate an explicit $\Sigma$-dependence [7].

What concerns the connection $\tilde{\Gamma}$ we confirmed Ivanov's result [18] that supersymmetry constraints, Bianchi identity, and equations of motion are compatible only for $\tilde{\Gamma}=\Gamma^{-}$, even in the case of $\mathrm{AdS}_{4}$ compactifications with a gaugino condensate. Even without supersymmetry, we found that the choice $\tilde{\Gamma}=\Gamma^{-}$is mandatory.

\section{Acknowledgments}

This work was partially supported by the Deutsche Forschungsgemeinschaft, the cluster of excellence QUEST, the Heisenberg-Landau program and RFBR grant 09-02-91347.

\section{References}

[1] A. Bachelot, The Dirac system on the Anti-de Sitter Universe, Commun. Math. Phys. 283 (2008) 127 [arXiv:0706.1315] [SPIRES].

[2] H. Baum, Twistor spinors on Lorentzian symmetric spaces, math.DG/9803089.

[3] K. Becker and S. Sethi, Torsional heterotic geometries, Nucl. Phys. B 820 (2009) 1 [arXiv: 0903.3769] [SPIRES].

[4] E.A. Bergshoeff and M. de Roo, The quartic effective action of the heterotic string and supersymmetry, Nucl. Phys. B 328 (1989) 439 [SPIRES].

[5] C. Bohle, Killing and twistor spinors on Lorentzian manifolds, http://www.math.tu-berlin.de/ bohle/pub/dipl.dvi.

[6] J.-B. Butruille, Homogeneous nearly Kähler manifolds, math/0612655.

[7] G. Lopes Cardoso, G. Curio, G. Dall'Agata and D. Lüst, Heterotic string theory on non-Kähler manifolds with H- flux and gaugino condensate, Fortsch. Phys. 52 (2004) 483 [hep-th/0310021] [SPIRES].

[8] G. Lopes Cardoso et al., Non-Kähler string backgrounds and their five torsion classes, Nucl. Phys. B 652 (2003) 5 [hep-th/0211118] [SPIRES].

[9] M. Fernandez, S. Ivanov, L. Ugarte and R. Villacampa, Non-Kähler heterotic string compactifications with non-zero fluxes and constant dilaton, Commun. Math. Phys. 288 (2009) 677 [arXiv:0804.1648] [SPIRES].

[10] A.R. Frey and M. Lippert, AdS strings with torsion: non-complex heterotic compactifications, Phys. Rev. D 72 (2005) 126001 [hep-th/0507202] [SPIRES].

[11] T. Friedrich, On types of non-integrable geometries, math/0205149.

[12] J.-X. Fu and S.-T. Yau, The theory of superstring with flux on non-Kähler manifolds and the complex Monge-Ampère equation, J. Diff. Geom. 78 (2009) 369 [hep-th/0604063] [SPIRES]. 
[13] T.R. Govindarajan, A.S. Joshipura, S.D. Rindani and U. Sarkar, Supersymmetric compactification of the heterotic string on coset spaces, Phys. Rev. Lett. 57 (1986) 2489 [SPIRES].

[14] M.B. Green, J.H. Schwarz and E. Witten, Superstring theory, Vol. 2: Loop amplitudes, anomalies $\&$ phenomenology, Cambridge University Press (1987).

[15] R. Grunewald, Six-dimensional Riemannian manifolds with a real Killing spinor,Ann. Global Anal. Geom. 8 (1990) 43.

[16] D. Harland, T.A. Ivanova, O. Lechtenfeld and A.D. Popov, Yang-Mills flows on nearly Kähler manifolds and $G_{2}$-instantons, arXiv:0909.2730 [SPIRES].

[17] C.M. Hull, Anomalies, ambiguities and superstrings, Phys. Lett. B 167 (1986) 51 [SPIRES].

[18] S. Ivanov, Heterotic supersymmetry, anomaly cancellation and equations of motion, Phys. Lett. B 685 (2010) 190 [arXiv:0908.2927] [SPIRES].

[19] S. Kobayashi and K. Nomizu, Foundations of differential geometry, Vol. 1, John Wiley \& Sons (1963).

[20] J. Li and S.-T. Yau, The existence of supersymmetric string theory with torsion, J. Diff. Geom. 70 (2005) 143 [hep-th/0411136] [SPIRES].

[21] P. Manousselis, N. Prezas and G. Zoupanos, Supersymmetric compactifications of heterotic strings with fluxes and condensates, Nucl. Phys. B 739 (2006) 85 [hep-th/0511122] [SPIRES].

[22] J. Polchinski, String theory, Vol. I, Cambridge University Press (2005).

[23] A.D. Popov, Hermitian-Yang-Mills equations and pseudo-holomorphic bundles on nearly Kähler and nearly Calabi-Yau twistor 6-manifolds, Nucl. Phys. B 828 (2010) 594 [arXiv: 0907.0106] [SPIRES].

[24] A. Strominger, Superstrings with torsion, Nucl. Phys. B 274 (1986) 253 [SPIRES].

[25] L. Ugarte and R. Villacampa, Non-nilpotent complex geometry of nilmanifolds and heterotic supersymmetry, arXiv:0912.5110. 